\title{
Stability and Bifurcation in Magnetic Flux Feedback Maglev Control System
}

\author{
Wen-Qing Zhang, Jie Li, Kun Zhang, and Peng Cui \\ College of Mechatronics Engineering and Automation, National University of Defense Technology, Changsha, Hunan 410073, China \\ Correspondence should be addressed to Wen-Qing Zhang; zwq197566@163.com
}

Received 1 June 2013; Accepted 7 August 2013

Academic Editor: Massimo Scalia

Copyright ( 2013 Wen-Qing Zhang et al. This is an open access article distributed under the Creative Commons Attribution License, which permits unrestricted use, distribution, and reproduction in any medium, provided the original work is properly cited.

\begin{abstract}
Nonlinear properties of magnetic flux feedback control system have been investigated mainly in this paper. We analyzed the influence of magnetic flux feedback control system on control property by time delay and interfering signal of acceleration. First of all, we have established maglev nonlinear model based on magnetic flux feedback and then discussed hopf bifurcation's condition caused by the acceleration's time delay. The critical value of delayed time is obtained. It is proved that the period solution exists in maglev control system and the stable condition has been got. We obtained the characteristic values by employing center manifold reduction theory and normal form method, which represent separately the direction of hopf bifurcation, the stability of the period solution, and the period of the period motion. Subsequently, we discussed the influence maglev system on stability of by acceleration's interfering signal and obtained the stable domain of interfering signal. Some experiments have been done on CMS04 maglev vehicle of National University of Defense Technology (NUDT) in Tangshan city. The results of experiments demonstrate that viewpoints of this paper are correct and scientific. When time lag reaches the critical value, maglev system will produce a supercritical hopf bifurcation which may cause unstable period motion.
\end{abstract}

\section{Introduction}

The maglev vehicle is a new traffic way. It has several advantages, including speeding up rapidly, less energy consumption, no noise, powerful braking effort, high riding quality, and minor radius of bending. So this traffic method will have more developments in short future. The maglev system carries out suspending function and is the core of the maglev train. Figure 1 gives its working principle. The guideway is flexible in engineering environment. Thus, the oscillation induced by Bernoulli-Euler beam equation must be considered. Dynamic response of maglev vehicle/guideway system has great influence on stability of the system. Maglev system is very complicated because it has a lot of uncertain factors and nonlinear coupling components. The resonance may appear when excitation frequency is close to hopf bifurcation frequency. Bifurcation phenomena happen in many industrial control domains. A method to locate bifurcations in time delay systems with a potentially high-dimensional parameter space has been denoted in the literature [1].
It can determine which parameters are relevant to complex dynamical behavior in such networks. Byrnes and Isidori analyze the bifurcation phenomenon [2] of the zero dynamics and the practical stabilization of nonlinear minimum-phase systems. Wang and Hill put forward a "deterministic learning (DL)" theory for identification of nonlinear system dynamics under full-state measurements. A systematic procedure for modeling and robust control of a multivariable magnetic levitation system is described in the literature [3] by scholars Tsujino et al. The discrete-time model of the magnetic levitation system [4] is derived and the stability is guaranteed by the root locus methodology. Banerjee et al. [5] design a control philosophy for simultaneous stabilization and performance improvement of an electromagnetic levitation system. And Shieh et al. have presented a robust optimal sliding-mode control approach [6] for position tracking of a magnetic levitation system. Ji et al. [7] apply an $H_{\infty}$ control to suppress the spillovers caused by unmodeled dynamics which we estimate using closed loop identification. A real-time operating environment [8] was established for closed-loop 


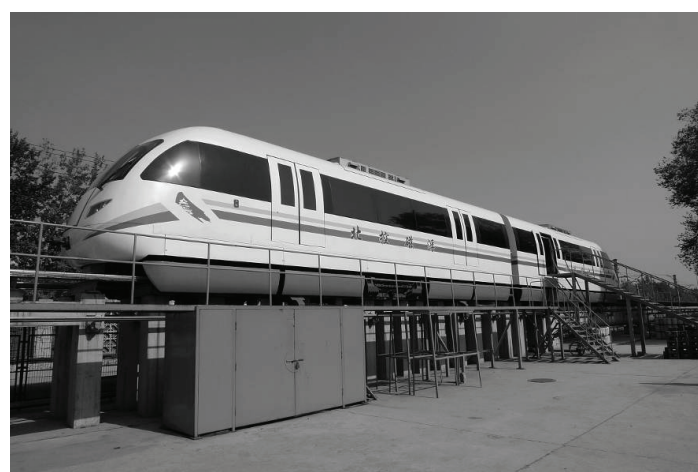

Figure 1: National mid-low speed maglev experimental field of Tangshan city.

control over Ethernet. Also, a novel discrete-time repetitive controller [9] design for time delay systems subject to a periodic reference and exogenous periodic disturbances is presented. Ariba et al. designed a new controller for firstorder linear time invariant system with time delay based on the Hermite-Biehler theorem [10]. In the paper [11], Ariba et al. propose an original approach: the quadratic separation. At the end of the paper, the delay operator properties are exploited to provide delay range stability conditions. The bifurcation phenomenon also appears in power system [12]. Ben-Kilani and Schlueter [13] denote that the bifurcation subsystem is a singular perturbation problem in fact, and this problem can be analyzed by the center manifold dynamics method. A local bifurcation of a parametrized differential equation occurs at an equilibrium where there is a change in the topological character of the nearby solution curves [14]. The unstable oscillation of autonomous dynamic system [15] of a matrix converter (MC) is studied based on nonlinear dynamic theory. Yang et al. prove that the eigenvalue crossing condition [16] for the hopf curve holds without additional assumption. Zhang and Jiang consider a delayed predatorprey system with Holling II functional response [17]. An effective hopf bifurcation criterion is provided for an induction motor (IM) drive system with indirect field oriented control (IFOC) [18]. The paper [19] deals with the problem of hopf bifurcation stabilization for Rössler system. Rössler system has two cures of equilibria, and hopf bifurcation may occur at some points of the equilibria. It is found that hopf bifurcation [20] occurs when these parameters pass through some critical values where the conditions for local asymptotical stability of the equilibrium are not satisfied. Guan and Chen [21] investigate the local hopf bifurcation in Genesio system with delayed feedback control. The time delay problem is one of the most significant issues [22]. The controller design [23] is cast into a convex multiobjective optimization problem with linear matrix inequality (LMI) constraints by defining a Lyapunov functional and using the delay partitioning method.

The main work of this paper is investigating the time delay of acceleration signal and the interference of acceleration in magnetic flux feedback maglev control system. We got

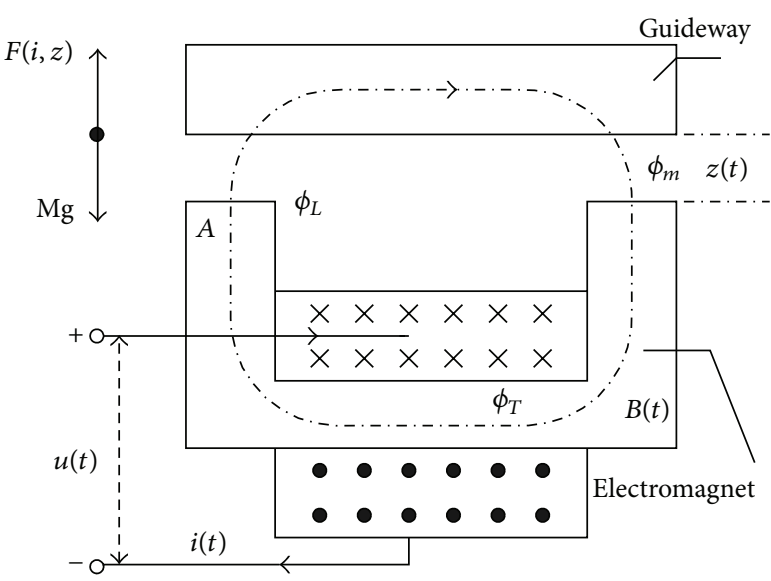

FIgURE 2: Simple model of maglev system.

characteristic values by employing the center manifold reduction and normal form theory, which represent separately the direction of hopf bifurcation, the stability of the period solution, and the period of the period motion. Maglev vehicle CMS04 has oscillation phenomenon because of time delay and interference. So the paper has studied the nature of maglev control system's period motion.

\section{Foundation of Magnetic Flux Feedback Suspension Control Model}

The maglev control model based on magnetic flux feedback is denoted in Figure 2.

Illuminate signs of Figure 2 in Table 1.

Assume the following.

(1) Magnetic flux formula $\phi_{T}=\phi_{L}+\phi_{m}$, neglect leaking flux, namely, $\phi_{L}=0$.

(2) Neglect magnetic resistance of iron core and guideway, and assume that magnetic field potential is distributed evenly on the suspension gap.

(3) Neglect deformation and elastic vibration of track, and track has infinite rigidity with regard to electromagnet.

The maglev control model has been founded based on flux feedback in the literature [12] already:

electromagnetics equation: $B=\frac{\mu_{0} N i(t)}{2 z(t)}$,

mechanics equation: $F=\frac{B^{2} A}{\mu_{0}}$,

kinematics equation: $M \ddot{z}(t)=-F+M g$,

electric equation: $u(t)=\frac{2 R z(t)}{\mu_{0} N} B(t)+N A \dot{B}(t)$.

The open loop block diagram of maglev control system is given in Figure 3. 


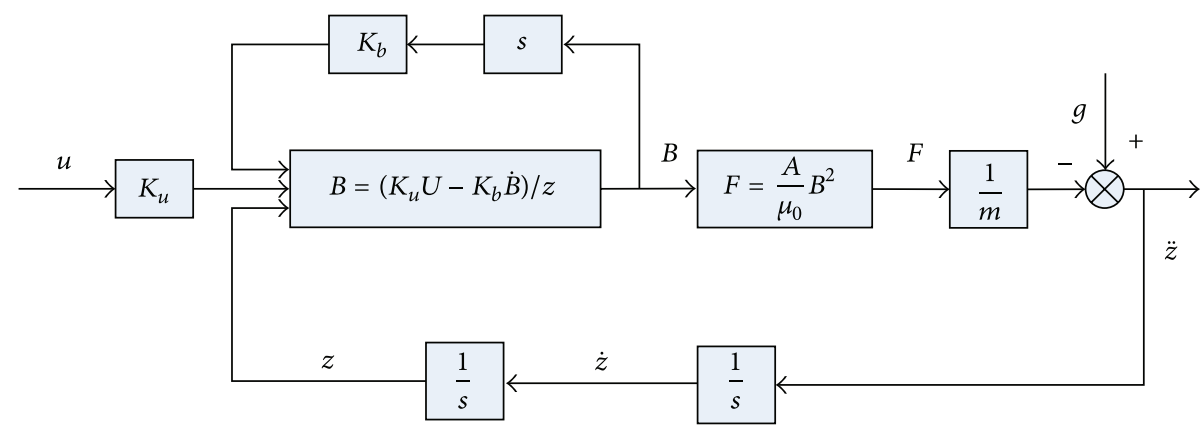

FIgURE 3: Open loop control block diagram of maglev system.

TABLE 1: Symbols of maglev system.

\begin{tabular}{ll}
\hline Signs & Significations \\
\hline$\phi_{m}$ & Gap flux \\
$\phi_{T}$ & Main flux \\
$\phi_{L}$ & Leaking flux \\
$\psi$ & Gap flux linkage \\
$u(t)$ & Voltage of magnetic coil \\
$W_{m}$ & Magnetic field energy in bulk V \\
$R$ & Resistance of magnetic coil \\
$B$ & Gap flux density \\
$\mu_{0}$ & Magnetic permeability of atmosphere \\
$F_{m}$ & Magnetic field potential \\
$R_{m}$ & Magnetic resistance \\
$A$ & Magnetic pole area \\
$i(t)$ & Current of magnetic coil \\
$w_{m}$ & Magnetic field energy density \\
$N$ & Number of magnetic coil \\
$z(t)$ & Gap among electromagnet and track \\
$F$ & Electromagnetic force \\
$M$ & Total mass \\
\hline
\end{tabular}

\section{Analysis of Nonlinear Property}

3.1. Stability and hopf Bifurcation. There are some reasons to cause time delay of acceleration signal, for example, signal transfer delay, the retardation between actuator and sensor, the filtering delay of acceleration signal, and so on. Suppose that delayed time is $\tau$, and acceleration signal after the delayed time is $\ddot{z}_{\tau}$; in this section, we will investigate stability and hopf bifurcation of the maglev system at the equilibrium. CMS04 generally applies PIDA control algorithm, and this method is represented in (2). When the system reaches stable status, the integral action can be neglected, so we simplify (2) to (3):

$$
\begin{gathered}
u(t)=K_{p} \cdot\left(z-z_{e}\right)+K_{i} \\
\cdot \int\left(z-z_{e}\right) d t+K_{d} \cdot \dot{z}+K_{a} \cdot \ddot{z}_{\tau} . \\
u(t)=K_{p}\left(z-z_{e}\right)+K_{d} \dot{z}+K_{a} \ddot{z}_{\tau} .
\end{gathered}
$$

According to (1), the control closed loop model based on flux feedback is given as

$$
\begin{gathered}
B=\sqrt{\frac{M \mu_{0}(g-\ddot{z})}{A},} \\
\dot{B}=-\frac{M \mu_{0}}{2 A \sqrt{M \mu_{0}(g-\ddot{z}) / A}} \ddot{z}, \\
u=\frac{2 R B z}{\mu_{0} N}+N A \dot{B}, \\
u=K_{p}\left(z-z_{e}\right)+K_{d} \dot{z}+K_{a} \ddot{z}_{\tau},
\end{gathered}
$$

where $z_{\tau}=z(t-\tau)$, and the closed loop equation of maglev system can be derived

$$
\begin{aligned}
\varphi^{\tau}(z, \dot{z}, \ddot{z}, \ddot{z}, \tau)= & \frac{M N \mu_{0}}{2 \sqrt{M \mu_{0}(g-\ddot{z}) / A}} \ddot{z} \\
& +K_{a} \ddot{z}_{\tau}+K_{d} \dot{z}+K_{p}\left(z+z_{e}\right) \\
& -\frac{2 R z}{\mu_{0} N} \sqrt{\frac{M \mu_{0}(g-\ddot{z})}{A}} .
\end{aligned}
$$

The working equilibrium point is $x_{0}=\left[\begin{array}{lll}z_{e} & 0 & 0\end{array}\right]^{T}$, and $z_{e}$ is the designed value of maglev system. Move the equilibrium point to the original point, and let $z=z-z_{e}$; then the closed loop equation of maglev system becomes

$$
\begin{aligned}
\varphi^{\tau}(z, \dot{z}, \ddot{z}, \ddot{z}, \tau)= & \frac{M N \mu_{0}}{2 \sqrt{M \mu_{0}(g-\ddot{z}) / A}} \ddot{z} \\
& +K_{a} \ddot{z}_{\tau}+K_{d} \dot{z}+K_{p} z \\
& -\frac{2 R\left(z+z_{e}\right)}{\mu_{0} N} \sqrt{\frac{M \mu_{0}(g-\ddot{z})}{A}} .
\end{aligned}
$$


Expand (6) up to third-order Taylor series about new equilibrium point $(0,0,0)$, and we obtain the following:

$$
\begin{aligned}
\varphi^{\tau}(z, \dot{z}, \ddot{z}, \ddot{z}, \tau) \\
=M_{1} \ddot{z}+\left(\frac{M M_{1} R z_{e}}{\mu_{0} A M N^{2}}+\frac{M_{1}}{2 g \mu_{0} M N}\right) \ddot{z} \\
\quad+K_{a} \ddot{z}_{\tau}+K_{d} \dot{z}+\left(K_{p}-\frac{R M_{1}}{A N^{2} \mu_{0}}\right) z \\
\quad+f\left(x, x_{\tau}\right)+O^{n, n \geq 3}(z, \dot{z}, \ddot{z}, \ddot{z}),
\end{aligned}
$$

where,

$$
\begin{aligned}
f\left(x, x_{\tau}\right)= & \left(\frac{3 M_{1}}{8 g^{2} M N \mu_{0}}+\frac{M R z_{e} M_{1}}{4 g N A M N \mu_{0}}\right) \ddot{z}^{2} \\
& -\frac{2 \mu_{0} M R z_{e}}{\mu_{0} M_{1}}, \\
M_{1}= & \frac{M N \mu_{0}}{\sqrt{M \mu_{0} g / A}} .
\end{aligned}
$$

Delete the nonlinear part, and get

$$
\begin{aligned}
\varphi_{0}^{\tau}(z, \dot{z}, \ddot{z}, \ddot{z}, \tau) \\
=M_{1} \dddot{z}+\left(\frac{M M_{1} R z_{e}}{\mu_{0} A M N^{2}}+\frac{M_{1}}{2 g \mu_{0} M N}\right) \ddot{z} \\
\quad+K_{a} \ddot{z}_{\tau}+K_{d} \dot{z}+\left(K_{p}-\frac{R M_{1}}{A N^{2} \mu_{0}}\right) z .
\end{aligned}
$$

We transform the time domain (9) into the frequency domain (10):

$$
\begin{aligned}
\varphi_{0}^{\tau}(s, \tau)= & M_{1} s^{3}+\left(\frac{M M_{1} R z_{e}}{\mu_{0} A M N^{2}}+\frac{M_{1}}{2 g \mu_{0} M N}\right) s^{2} \\
& +K_{a} s^{2} e^{-s \tau}+K_{d} s^{1}+\left(K_{p}-\frac{R M_{1}}{A N^{2} \mu_{0}}\right) s^{0} .
\end{aligned}
$$

Set $x=\left[\begin{array}{lll}z & \dot{z} & \ddot{z}\end{array}\right]^{T}, x_{\tau}=\left[\begin{array}{lll}z_{\tau} & \dot{z}_{\tau} & \ddot{z}_{\tau}\end{array}\right]^{T}$, and maglev linear model can be got at new equilibrium point:

$$
\begin{gathered}
\dot{x}(t)=A_{1} x(t)+A_{2} x_{\tau}(t)+f\left(x, x_{\tau}\right), \\
y(t)=C_{1} x(t)+C_{2} x_{\tau}(t),
\end{gathered}
$$

where

$$
\begin{gathered}
A_{1}=\left[\begin{array}{ccc}
0 & 1 & 0 \\
0 & 0 & 1 \\
-a_{3} & -a_{2} & -a_{1}
\end{array}\right], \quad A_{2}=\left[\begin{array}{ccc}
0 & 0 & 0 \\
0 & 0 & 0 \\
0 & 0 & -a_{1 \tau}
\end{array}\right], \\
C_{1}=\left[\begin{array}{lll}
1 & 0 & 0
\end{array}\right], \quad C_{2}=\left[\begin{array}{lll}
0 & 0 & 0
\end{array}\right], \\
a_{1}=M_{a}, \quad a_{1 \tau}=\frac{K_{a}}{M_{1}}, \quad a_{2}=\frac{K_{d}}{M_{1}} \\
a_{3}=\frac{\left(K_{p}-\left(R M_{1} / A N^{2} \mu_{0}\right)\right)}{M_{1}} \\
M_{a}=\frac{M R z_{e}}{\mu_{0} A M N^{2}}+\frac{1}{2 g \mu_{0} M N}
\end{gathered}
$$

Let $\varphi_{0}(z, \dot{z}, \ddot{z}, \ddot{z}, \tau)=0$, and the characteristic equation of maglev system changes as follows:

$$
\lambda^{3}+a_{1} \lambda^{2}+a_{2} \lambda^{1}+a_{3} \lambda^{0}+a_{1 \tau} \lambda^{2} \exp (-\lambda \tau)=0
$$

All properties of the closed loop control system are determined by distributing roots of the previously determined equation. If maglev control system keeps local stability at the equilibrium point $(0,0,0)$, it must insure that roots of the characteristic equation all have negative real part:

$$
a_{1}>0, \quad\left(a_{1}+a_{1 \tau}\right) a_{2}-a_{3}>0, \quad a_{3}>0
$$

When time lag $\tau>0$, only if it becomes small enough, the above condition can keep the characteristic equation have negative real part, or else the above condition cannot keep that the system's trivial solution has local stability. Because the roots change sequentially, and when $a_{3} \neq 0$, zero is not root of the characteristic equation, so if bifurcation occurs, it must be a dynamic hopf bifurcation. When time lag increases close to the critical value, a pair of conjugate pure imaginary roots will appear in characteristic equation, and other roots all have the negative real part. Assume that the pair of conjugate pure imaginary roots are $\lambda= \pm i w(w>0)$, and substitute them into the characteristic equation:

$$
(i w)^{3}+a_{1}(i w)^{2}+a_{2}(i w)^{1}+a_{3}(i w)^{0}+a_{1 \tau}(i w)^{2} e^{-i w \tau}=0
$$

Separate the real part and the imaginary part, and get

$$
\gamma(w) \pm i \eta(w)=0
$$

where

$$
\begin{aligned}
& \gamma(w)=a_{3}-a_{1} w^{2}-a_{1 \tau} w^{2} \cos (w \tau), \\
& \eta(w)=a_{2} w-w^{3}+a_{1 \tau} w^{2} \sin (w \tau) .
\end{aligned}
$$

Eliminate $\sin (w \tau)$ and $\cos (w \tau)$ from the above equation, and we can obtain

$$
w^{6}+\left(a_{1}^{2}-2 a_{2}\right) w^{4}+\left(a_{2}^{2}+2 a_{1} a_{3}\right) w^{2}+a_{3}^{2}-a_{1 \tau}^{2} w^{4}=0 .
$$

If (18) has no positive real root, the stability of the system will have no change with the time delay varying. If it has a positive real root, then put it into the following equation:

$$
\begin{aligned}
& \sin (w \tau)=\frac{w^{3}-a_{2} w}{a_{1 \tau} w^{2}}, \\
& \cos (w \tau)=\frac{a_{3}-a_{1} w^{2}}{a_{1 \tau} w^{2}} .
\end{aligned}
$$

The minimal time lag obtained is critical value $\tau_{0}$, and the corresponding value of $w$ is $w_{0}$. hopf bifurcation happens at 
the moment. The differential equation of formula (13) is as follows:

$$
\begin{gathered}
{\left[a_{2}-3 w_{0}^{2}+2 a_{1 \tau} w_{0} \sin \left(w_{0} \tau_{0}\right)+a_{1 \tau} \tau_{0} w_{0}^{2} \cos \left(w_{0} \tau_{0}\right)\right] d \lambda} \\
+\left[2 a_{1} w_{0}+2 a_{1 \tau} w_{0} \cos \left(w_{0} \tau_{0}\right)\right. \\
\left.\quad-a_{1 \tau} \tau_{0} w_{0}^{2} \sin \left(w_{0} \tau_{0}\right)\right] i d \lambda \\
=a_{1 \tau} w_{0}^{3} \sin \left(w_{0} \tau_{0}\right) d \tau-a_{1 \tau} w_{0}^{3} \cos \left(w_{0} \tau_{0}\right) i d \tau \\
\operatorname{Re}\left(\frac{d \lambda}{d \tau}\right)_{\tau=\tau_{0}}^{\lambda=i w_{0}}=\frac{a_{1 \tau} w_{0}^{3} \sin \left(w_{0} \tau_{0}\right)}{q_{11}^{2}+q_{12}^{2}}
\end{gathered}
$$

where the terms

$$
\begin{gathered}
q_{11}=a_{2}-3 w_{0}^{2}+2 a_{1 \tau} w_{0} \sin \left(w_{0} \tau_{0}\right)+a_{1 \tau} \tau_{0} w_{0}^{2} \cos \left(w_{0} \tau_{0}\right) \\
q_{12}=2 a_{1} w_{0}+2 a_{1 \tau} w_{0} \cos \left(w_{0} \tau_{0}\right)-a_{1 \tau} \tau_{0} w_{0}^{2} \sin \left(w_{0} \tau_{0}\right) .
\end{gathered}
$$

If $\operatorname{Re}(d \lambda / d \tau)_{\tau=\tau_{0}}^{\lambda=i w_{0}} \neq 0$, then a pair of complex eigenvalues crosses the imaginary axis with the time lag changing; at the end the hopf bifurcation will occur.

3.2. Orientation of hopf Bifurcation and Stability of Periodic Solution. The critical point of hopf bifurcation has been discussed in front section. We will investigate the stability of periodic solution of hopf bifurcation by employing center manifold reduction and normal form theory in this section.

Set $\tau=\tau_{0}+\mu$, and the phase space of the maglev closed loop control system is $C=C\left(\left[-\tau_{0}, 0\right], R^{3}\right)$, so the characteristic equation can be transformed into a function differential equation as follows:

$$
\dot{x}_{t}=D_{\mu} x_{t}+f\left(x_{t}, \mu\right), \quad x_{t} \in R^{3}, t>0,
$$

where $x_{t}(\theta)=x(t+\theta), \theta \in\left[-\tau_{0}, 0\right]$, and $D_{\mu} x_{t}, f\left(x_{t}, \mu\right)$ separately are linear part and nonlinear part. The terms $D_{\mu}$ : $C \rightarrow C$ and $f: C \rightarrow C$ are defined as

$$
\begin{gathered}
D_{\mu} \Phi=A_{1} \Phi(0)+\left(\tau_{0}+\mu\right) A_{2} \Phi\left(-\tau_{0}\right), \\
f(\mu, \Phi)=\left(\tau_{0}+\mu\right)\left(\begin{array}{c}
0 \\
0 \\
f\left(x, x_{\tau}\right)
\end{array}\right) .
\end{gathered}
$$

There is a bounded function $\gamma(\mu, \theta)$, based on Riesz representation theorem. We obtain $\theta \in\left[-\tau_{0}, 0\right]$ :

$$
D_{\mu} \Phi=\int_{-\tau_{0}}^{0} d \gamma(\mu, \theta) \Phi(\theta), \quad \Phi \in C,
$$

where

$$
\gamma(\mu, \theta)=A_{1} \delta(\theta)-\left(\tau_{0}+\mu\right) A_{2} \delta\left(\theta+\tau_{0}\right),
$$

and $\delta(\theta)$ is an impulse function.
For any $\Phi \in C^{1}\left(\left[-\tau_{0}, 0\right], R^{3}\right)$, define the adjoint operators $E$ and $E^{*}$ :

$$
\begin{gathered}
E(\mu) \Phi= \begin{cases}\frac{d \Phi(\theta)}{d \theta}, & \theta \in\left[-\tau_{0}, 0\right), \\
\int_{-\tau_{0}}^{0} d \gamma(\mu, \xi) \Phi(\xi), & \theta=0,\end{cases} \\
F(\mu) \Phi= \begin{cases}0, & \theta \in\left[-\tau_{0}, 0\right), \\
f(\mu, \Phi), & \theta=0 .\end{cases}
\end{gathered}
$$

Equation (23) can be written as $\dot{x}_{t}=E(\mu) x_{t}+F(\mu) x_{t}$, according to $d x_{t} / d \theta=d x_{t} / d t$ and the definition before.

The conjugate operator can be defined as follows for any $\psi \in C^{1}\left(\left[-\tau_{0}, 0\right],\left(R^{3}\right)^{*}\right)$.

$$
E(0) \Phi(\theta)= \begin{cases}\frac{-d \Phi(\theta)}{d \theta} & \theta \in\left(0, \tau_{0}\right], \\ \int_{-\tau_{0}}^{0} d \gamma(\theta, 0) \Phi(-\theta), & \theta=0 .\end{cases}
$$

The inner product of the vector $\psi$ and $\Phi$ is

$$
\begin{aligned}
\langle\psi(\theta), \Phi(\theta)\rangle= & \bar{\psi}(0) \Phi(0) \\
& -\int_{-\tau_{0}}^{0} \int_{\xi=0}^{0} \bar{\psi}(\xi-\theta) d \gamma(\theta) \Phi(\xi) d \xi,
\end{aligned}
$$

where $\bar{\psi}$ denotes the complex conjugation operation for transposition of the vector $\psi$.

Proof of the Periodic Solution and hopf Bifurcation's Stability

Step 1. Suppose that the operators $E$ and $E^{*}$ are the eigenvectors corresponding to the eigenvalues $\pm i w_{0} \tau_{0}$ and satisfy

$$
\begin{gathered}
p(\theta)=\left[\begin{array}{lll}
1 & \alpha & \beta
\end{array}\right]^{T} e^{i w_{0} \tau_{0} \theta}, \\
p^{*}(\theta)=H\left[\begin{array}{lll}
1 & \alpha & \beta
\end{array}\right]^{T} e^{i w_{0} \tau_{0} \theta} .
\end{gathered}
$$

According to (24), yield

$$
\begin{gathered}
E p(\theta)=i w_{0} \tau_{0} \theta p(\theta), \\
E^{*} p^{*}(\theta)=-i w_{0} \tau_{0} \theta p^{*}(\theta) .
\end{gathered}
$$

The solutions can be obtained

$$
\begin{gathered}
\alpha=\frac{a_{1 \tau} w_{0} \cos \left(w_{0} \tau_{0}\right)-\left(a_{1}+1\right) w_{0}-a_{3}}{\left(a_{2}+1\right) a_{1 \tau} \sin \left(w_{0} \tau_{0}\right)}, \\
\beta=\frac{w_{0}}{a_{1 \tau} \sin \left(w_{0} \tau_{0}\right)}, \\
\alpha_{1}=\frac{\left(a_{1}+i w_{0}-a_{1 \tau} e^{i w_{0}}\right)\left(1-i w_{0}\right)}{\left(1-i w_{0}\right)\left(a_{2}+i w_{0}\right)}, \\
\beta_{1}=\frac{1-i w_{0}}{a_{2}+i w_{0}} .
\end{gathered}
$$

$q(\theta)$ and $q^{*}(\theta)$ can be solved according to orthonormality conditions $\left\langle q^{*}(\theta), q(\theta)\right\rangle=1,\left\langle q^{*}(\theta), \bar{q}(\theta)\right\rangle=0$, and we will get

$$
H=\frac{1}{1+\bar{\alpha} \alpha_{1}+\bar{\beta} \beta_{1}} .
$$


Step 2. Introduce new variables $z(t)=\left\langle q^{*}, x_{t}\right\rangle, W(t, \theta)=$ $x_{t}(\theta)-2 \operatorname{Re}\{z(t) q(\theta)\}$, and the Poincaré normal form of (20) can be written as

$$
W(t, \theta)=W(z(t), \bar{z}(t), \theta),
$$

$$
\begin{aligned}
W(z(t), \bar{z}(t), \theta)= & W_{20}(\theta) \frac{z^{2}}{2}+W_{11}(\theta) z \bar{z} \\
& +W_{02}(\theta) \frac{\bar{z}^{2}}{2}+W_{30}(\theta) \frac{z^{3}}{2}+\cdots
\end{aligned}
$$

The plane spanned by eigenvectors $z$ and $\bar{z}$ is tangent to center manifold at the origin. This means that center manifold can be locally approximated as a truncated power series of $W$ depending on the second order of the coordinates $z$ and $\bar{z}$.

Step 3. Only consider real solution. When $\mu=0$, the solution of (20) is $x_{t} \in C_{0}$. According to the above equations, get

$$
\begin{aligned}
\dot{z}(t) & =\left\langle q^{*}, \dot{x}_{t}\right\rangle=\left\langle q^{*}, E \dot{x}_{t}+F x\right\rangle \\
& =i w_{0} \tau_{0} z(t)+\bar{q}^{*}(0) f_{0}(z, \bar{z}) \\
& =i w_{0} \tau_{0} z(t)+k(z, \bar{z}), \\
f_{0}(z, \bar{z}) & =f\left(0, x_{t}(\theta)\right) \\
& =f(0, W(z, \bar{z}, \theta))+2 \operatorname{Re}\{z q(\theta)\} .
\end{aligned}
$$

Define

$$
\begin{aligned}
& g(z, \bar{z})=\bar{q}^{*}(0) f_{0}(z, \bar{z}) \\
& =k_{20}(\theta) \frac{z^{2}}{2}+k_{11}(\theta) z \bar{z}+k_{02}(\theta) \frac{\bar{z}^{2}}{2} \\
& +k_{30}(\theta) \frac{z^{2} \bar{z}}{2}+\cdots, \\
& x_{t}(\theta)=W(t, \theta)+2 \operatorname{Re}\{z q(\theta)\} \\
& =k_{20}(\theta) \frac{z^{2}}{2}+k_{11}(\theta) z \bar{z}+k_{02}(\theta) \frac{\bar{z}^{2}}{2} \\
& +\left(\begin{array}{lll}
1 & \alpha & \beta
\end{array}\right)^{T} e^{i w_{0} \tau_{0} \theta} z+\left(\begin{array}{lll}
1 & \bar{\alpha} & \bar{\beta}
\end{array}\right)^{T} e^{-i w_{0} \tau_{0} \theta} \bar{z}+\cdots \text {. }
\end{aligned}
$$

The values of $k_{20}, k_{02}, k_{11}$, and $k_{21}$ can be calculated by (35) and (36).

Step 4. Calculate $W_{20}(\theta)$ and $W_{11}(\theta)$

$$
\begin{aligned}
\dot{W} & =\dot{x}_{t}-\dot{z} p-\dot{\bar{z}} \bar{p} \\
& = \begin{cases}E W-2 \operatorname{Re}\left\{\bar{p}^{*}(0) f_{0}(z, \bar{z}) p(\theta)\right\}, & \theta \in\left[-\tau_{0}, 0\right), \\
E W-2 \operatorname{Re}\left\{\bar{p}^{*}(0) f_{0}(z, \bar{z}) p(0)\right\}, & \theta=0,\end{cases}
\end{aligned}
$$

where

$$
\begin{aligned}
M(z, \bar{z}, \theta)= & M_{20}(\theta) \frac{z^{2}}{2}+M_{11}(\theta) z \bar{z} \\
& +M_{02}(\theta) \frac{\bar{z}^{2}}{2}+M_{30}(\theta) \frac{z^{3}}{6}+\cdots
\end{aligned}
$$

Differentiating $M$ from $t$, yield

$$
\dot{M}=M_{z} \dot{z}+M_{\bar{z}} \dot{\bar{z}}
$$

Further, yield

$$
\begin{gathered}
H_{20}(\theta)=W_{20}(\theta)\left(2 i w_{0}-A_{0}\right), \\
H_{11}(\theta)=-A_{0} W_{11}(\theta), \\
H_{02}(\theta)=-W_{02}(\theta)\left(A_{0}+2 i w_{0}\right) .
\end{gathered}
$$

According to the above equations and $\theta \in\left[-\tau_{0}^{*}\right)$, we can obtain

$$
\begin{aligned}
M(z, \bar{z}, \theta)= & -2 \operatorname{Re}\left\{\bar{q}^{*}(0) f_{0}(z, \bar{z}) p(\theta)\right\} \\
= & -k(z, \bar{z}) p(\theta)-\bar{k}(z, \bar{z}) \bar{p}(\theta) \\
= & -\left(\frac{1}{2} k_{20} z^{2}+k_{11} z \bar{z}+\frac{1}{2} k_{02} \bar{z}^{2}+\cdots\right) p(\theta) \\
& -\left(\frac{1}{2} k_{20} \bar{z}^{2}+k_{11} z \bar{z}+\frac{1}{2} k_{02} z^{2}+\cdots\right) \bar{p}(\theta) .
\end{aligned}
$$

Compare coefficients of (36) and (39), and obtain

$$
\begin{aligned}
& M_{20}(\theta)=-k_{20} p(\theta)-\bar{k}_{02} \bar{p}(\theta), \\
& M_{11}(\theta)=-k_{11} p(\theta)-\bar{k}_{11} \bar{p}(\theta) .
\end{aligned}
$$

Further

$$
\begin{aligned}
& \dot{W}_{20}(\theta)=2 i w_{0} W_{20}(\theta)+k_{20} p(\theta)+\bar{k}_{02} \bar{p}(\theta), \\
& \dot{W}_{11}(\theta)=k_{11} p(\theta)+\bar{k}_{11} \bar{p}(\theta) .
\end{aligned}
$$

The solution of (43) is

$$
\begin{aligned}
& W_{20}(\theta)=-\frac{k_{20} p(0)}{i w_{0}} e^{i w_{0} \theta}-\frac{\bar{k}_{02} \bar{p}(0)}{3 i w_{0}} e^{-i w_{0} \theta}+R_{1} e^{2 i w_{0} \theta}, \\
& W_{20}(\theta)=-\frac{k_{11} p(0)}{i w_{0}} e^{i w_{0} \theta}-\frac{\bar{k}_{02} \bar{p}(0)}{i w_{0}} e^{-i w_{0} \theta}+R_{2},
\end{aligned}
$$

where $R_{1}$ and $R_{2}$ are constant vectors which can be determined by setting $\theta=0$ in $H(z, \bar{z}, \theta)$.

Step 5. Calculate the characteristic quantity $\beta_{2}$ of the stability of hopf bifurcation, the characteristic quantity $\mu_{2}$ of the direction of bifurcation, and the characteristic quantity $T_{2}$ of the periodic variation. Before the calculation, we need to obtain canonical form coefficient $c_{1}(0)$ :

$$
\begin{gathered}
c_{1}(0)=\frac{i}{2 w_{0} \tau_{0}}\left(k_{20} k_{11}-2\left|k_{11}\right|^{2}-\frac{1}{3}\left|k_{02}\right|^{2}\right)+\frac{1}{2} k_{21}, \\
\mu_{2}=\frac{\operatorname{Re}\left\{c_{1}(0)\right\}}{\operatorname{Re}\left\{\lambda^{\prime}\left(\tau_{0}\right)\right\}}, \\
\nu_{2}=2 \operatorname{Re}\left\{c_{1}(0)\right\}, \\
T_{2}=-\frac{1}{w_{0} \tau_{0}}\left[\operatorname{Im}\left\{c_{1}(0)\right\}+\mu_{2} \operatorname{Im}\left\{\lambda^{\prime}\left(\tau_{0}\right)\right\}\right] .
\end{gathered}
$$


Step 6. To sum up, we get the theorem and it is represented as follows.

Theorem 1. Suppose $f_{i}, k_{i} \in C^{3}, i=1,2,3$; then the direction of hopf bifurcation can be determined by $\mu_{2}$. If $\mu_{2}>0\left(\mu_{2}<\right.$ 0 ), hopf bifurcation is supercritical (subcritical). When time lag $\tau>\tau_{0}\left(\tau<\tau_{0}\right)$, the periodic solution of bifurcation is stable (unstable). $T_{2}$ can determine the periodic law of bifurcation: if $T_{2}>0\left(T_{2}<0\right)$, the periodic solution will crease (decrease). Otherwise, the period of periodic motion can be evaluated by

$$
T(\mu)=\frac{2 \pi}{w_{0}}\left(1+T_{2} \mu^{2}+\cdots\right)
$$

\section{Stability and Interference}

We find disturbing signal of acceleration can be defined as $\ddot{z}_{\sigma}=\left(\sigma^{n}+1\right) \cdot \ddot{z}$, through a number of maglev experiments on the vehicle CMS04. The term $n$ is the power of disturbing signal. The positive direction is equal to the direction of gravity acceleration. So suppose disturbing model of acceleration is $p(x)=\left[\begin{array}{lll}0 & 0 & \sigma^{n}\end{array}\right] x$, and then the characteristic equation of maglev system about $\sigma$ is

$$
\begin{aligned}
\varphi_{0}^{\sigma}(z, \dot{z}, \ddot{z}, \dddot{z}, \sigma) \\
=M_{1} \dddot{z}+\left(\frac{M M_{1} R z_{e}}{\mu_{0} A M N^{2}}+\frac{M_{1}}{2 g \mu_{0} M N}+K_{a}\left(1+\sigma^{n}\right)\right) \ddot{z} \\
\quad+K_{d} \dot{z}+\left(K_{p}-\frac{R M_{1}}{A N^{2} \mu_{0}}\right) z .
\end{aligned}
$$

Simplify the characteristic equation, and get

$$
\lambda^{3}+a_{1}^{\sigma} \lambda^{2}+a_{2}^{\sigma} \lambda^{1}+a_{3}^{\sigma}=0
$$

where

$$
\begin{gathered}
a_{1}^{\sigma}=\frac{M R z_{e}}{\mu_{0} A M N^{2}}+\frac{1}{2 g \mu_{0} M N}+\frac{K_{a}\left(1+\sigma^{n}\right)}{M_{1}}, \\
a_{2}^{\sigma}=\frac{K_{d}}{M_{1}}, \quad a_{3}^{\sigma}=\frac{K_{p}}{M_{1}}-\frac{R}{A N^{2} \mu_{0}} .
\end{gathered}
$$

Let $\varphi_{0}^{\sigma}(z, \dot{z}, \ddot{z}, \dddot{z}, \sigma)=0$; then

$$
\lambda^{3}+a_{1}^{\sigma} \lambda^{2}+a_{2}^{\sigma} \lambda^{1}+a_{3}^{\sigma}=0
$$

Namely,

$$
\lambda^{3}+\left(a_{1}+\frac{K_{a}}{M_{1}} \sigma^{n}\right) \lambda^{2}+a_{2} \lambda^{1}+a_{3}=0
$$

The stability of system about $\sigma$ should be discussed at once.
According to Routh criterion, the condition of maglev system stability must be satisfied: all of eigenvalues must be positive and satisfy

$$
\begin{gathered}
D_{1}=a_{1}+\frac{K_{a}}{M_{1}} \sigma^{n}>0, \\
D_{2}=\left|\begin{array}{cc}
a_{1}+\frac{K_{a}}{M_{1}} \sigma^{n} & a_{3} \\
1 & a_{2}
\end{array}\right|>0, \\
D_{3}=a_{3} D_{2}>0 .
\end{gathered}
$$

The above equation can be simplified to

$$
\sigma^{n}>\left(\frac{a_{3}}{a_{2}}-a_{1}\right) \frac{K_{a}}{M_{1}},
$$

where

$$
\left(\frac{a_{3}}{a_{2}}-a_{1}\right) K_{a}<0
$$

according to the stable condition of the original system: $\left(a_{3} / a_{2}\right)-a_{1}<0, K_{a}>0$.

In summary, if the additive interfering signal of acceleration is positive, it cannot influence stability of maglev system. Through the experiments, this view has been proved and the additive positive interference can improve stability of system improve, on the contrary, because the positive interference changes the poles of maglev system far from the origin. Similarly, the positive multiplicative interference cannot influence stability of the system.

Discuss how the positive additive disturbs stability of maglev system

$$
\lambda^{3}+\left(a_{1}-\frac{K_{a}}{M_{1}} \sigma^{n}\right) \lambda^{2}+a_{2} \lambda^{1}+a_{3}=0 .
$$

According to Routh criterion, the system must satisfy (55) for keeping itself asymptotically stable

$$
\sigma^{n}<\min \left(\frac{M_{1} a_{1}}{K_{a}}, \frac{M_{1}\left(a_{1}-\left(a_{3} / a_{2}\right)\right)}{K_{a}}\right)=\frac{\left(a_{1} a_{2}-a_{3}\right) M_{1}}{a_{2} K_{a}} .
$$

If the negative interfering signal of acceleration reaches the critical condition, the maglev control system will change from stability to divergence. If the gain of interfering signal belongs to the set $U_{\sigma}^{s}=\left\{\sigma \mid \sigma^{n}>\left(a_{3}-a_{1} a_{2}\right) M_{1} / a_{2} K_{a}\right\}$, maglev system will be asymptotic stability. If the gain of interfering signal belongs to the set $U_{\sigma}^{u}=\left\{\sigma \mid \sigma^{n}<\left(a_{3}-\right.\right.$ $\left.\left.a_{1} a_{2}\right) M_{1} / a_{2} K_{a}\right\}$, maglev system will be divergence, and the term $\sigma=\left(\left(a_{3}-a_{1} a_{2}\right) M_{1} / a_{2} K_{a}\right)^{1 / n}$.

\section{Experiments}

Some experiments have been implemented on CMS04 maglev control experiment platform designed by NUDT. Maglev control system applies position and flux double cascade control algorithm, the outer loop adopting PID control 


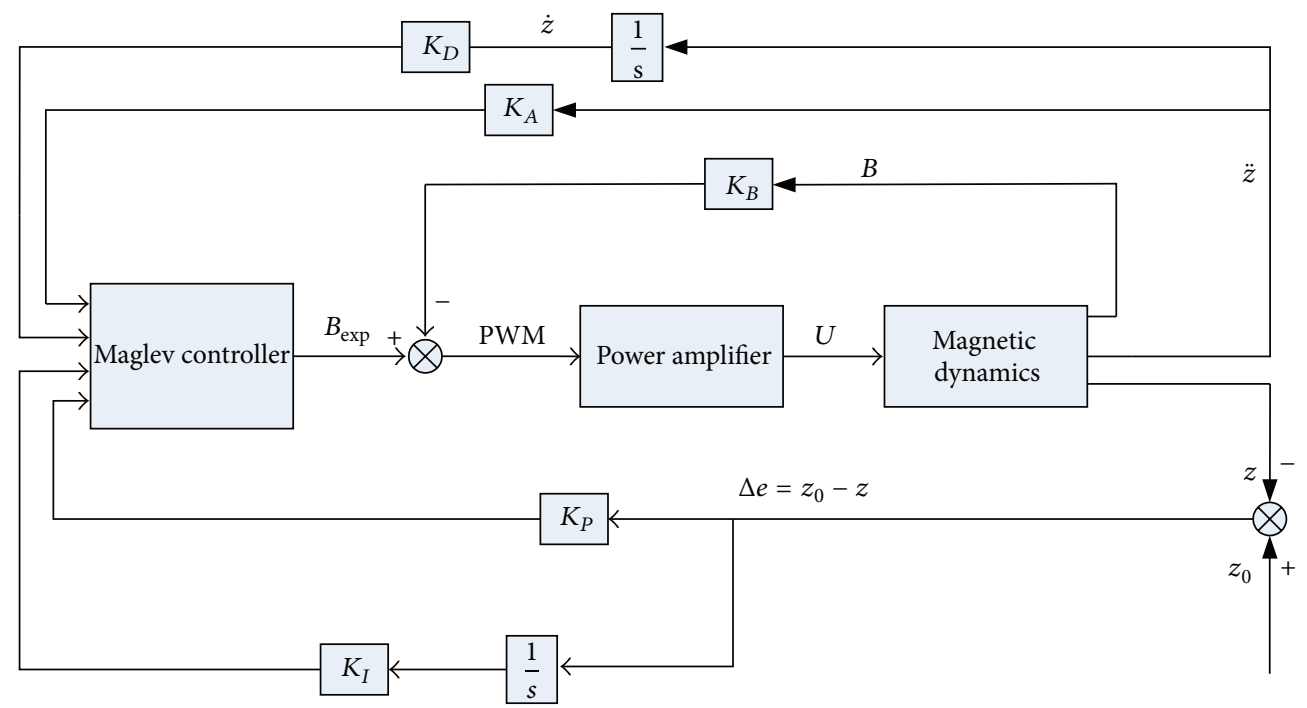

FIGURE 4: Block diagram of maglev closed control system.

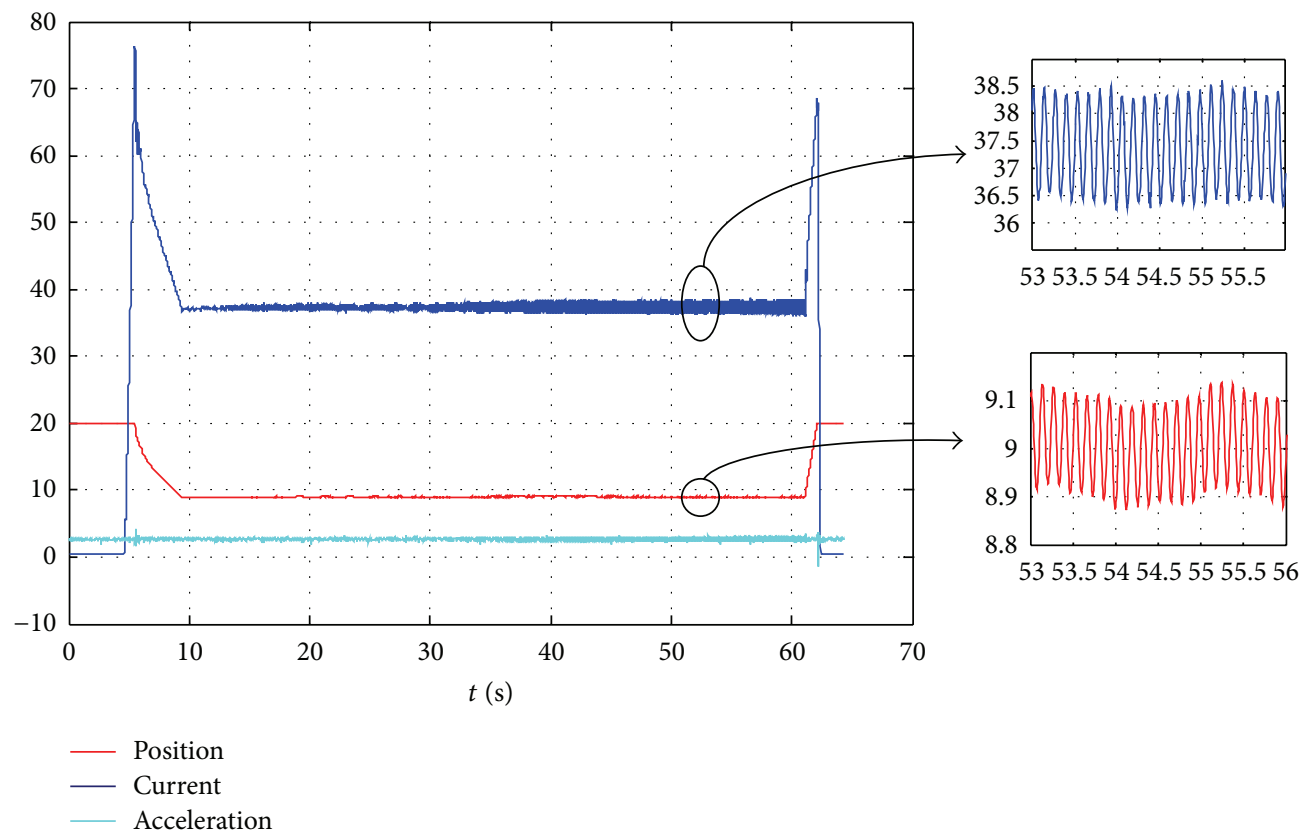

FIgURE 5: Position and current curves when $\tau=0.62$.

method, and the inner loop adopting $P$ control method. There is a detailed presentation about this control algorithm in the literature [12], so it is not stated in this paper. Some experiments have been done in terms of the theoretical result in this paper. The control parameters are shown in Table 2.

The maglev closed control system's block diagram is shown in Figure 4.

The control parameters in experiments are

$$
\begin{gathered}
K_{P}=4500, \quad K_{I}=4000, \quad K_{D}=800, \\
K_{A}=2, \quad K_{B}=60 .
\end{gathered}
$$

Experiment 1 (time delay of acceleration). The control parameters designed in experiment satisfy (11); namely, when $\tau=0$, the system is asymptotic stability. We obtain the values $w_{0}=2.1465$ and $\tau_{0}=0.6208$ from (45). Put the critical value into (21), and yield $\operatorname{Re}[d \lambda / d \tau]_{\tau=\tau_{0}}^{\lambda=i w_{0}}>0$; namely, it satisfies the crossing condition. When time lag gets close to $\tau_{0}$, hopf bifurcation will appear. Set delayed time $\tau=0.62 \mathrm{~s}$, and the solutions $\beta_{2} \approx 0.0432>0, \mu_{2} \approx-0.0371<0$, $T_{2} \approx 3.897>0$, which are calculated according to the theory of this paper. So we can diagnose that hopf bifurcation of maglev system is supercritical; namely, when delayed time is greater than $\tau_{0}$, hopf bifurcation happens, and the periodic solution of bifurcation is unstable, and the period increases gradually. When $\tau=0.62$, the response of maglev system is shown in Figure 5. The response proves that period motion 
TABLE 2: Standard maglev bogie's parameters of CMS04 maglev vehicle.

\begin{tabular}{lccc}
\hline Parameters & Values & Parameters & Values \\
\hline$M / \mathrm{kg}$ & 653 & $z_{0} / \mathrm{m}$ & 0.009 \\
$N$ & 324 & $B_{0} / \mathrm{T}$ & 0.6 \\
$A / \mathrm{m}^{2}$ & 0.0235 & $g$ & 9.8 \\
$R / \Omega$ & 0.5 & $\mu_{0}$ & $4 \pi \times 10^{-7}$ \\
\hline
\end{tabular}

appears in maglev control system, and the system's manifold will be divergent generally, and the phenomenon is that the suspension system has been vibrating consistently until divergence.

\section{Conclusions}

This paper has discussed hopf bifurcation caused by time delay of acceleration and denoted that when delayed time is close to the critical value $\tau_{0}$, hopf bifurcation will appear. This paper also obtains characteristic values by employing the center manifold reduction theory and the normal form method, which represent separately the direction of hopf bifurcation, the stability of period solution, and the period of period motion. Subsequently, we discuss the influence on the stability of maglev system by acceleration's interfering signal and obtain the stable domain of the interfering signal. Some experiments have been done on CMSO4 maglev vehicle by NUDT of Tangshan city. The results of experiments demonstrate that viewpoints in this paper are correct and scientific. When time lag reaches the critical value, maglev system will produce a supercritical hopf bifurcation which may cause unstable period motion. Therefore, the control engineers should decrease the retarded time between actuator and sensor and augment the accuracy of the suspending guideway with a view to make delayed time less than the critical value and make the control system escape from unstable period motion. The entire analysis illustrates that maglev system has complicated dynamic attribute, so this paper put more important references for investigating the dynamic property of maglev system further.

\section{Acknowledgment}

This work was financially supported by The National Natural Science Foundation of China (NNNSFC, nos. 11202230 and 60404003).

\section{References}

[1] S. Waldherr, D. Dylus, and F. Allgöwer, "Bifurcation search via feedback loop breaking in biochemical signaling pathways with time delay," Asian Journal of Control, vol. 13, no. 5, pp. 691-700, 2011.

[2] C. I. Byrnes and A. Isidori, "Bifurcation analysis of the zero dynamics and the practical stabilization of nonlinear minimum-phase systems," Asian Journal of Control, vol. 4, no. 2, pp. 171-185, 2002.
[3] T. Tsujino, K. Nakashima, and T. Fujii, "Application of $H_{\infty}$ control and closed loop identification to a magnetic levitation system," Asian Journal of Control, vol. 1, no. 4, pp. 283-296, 1999.

[4] J.-H. Li, "Fuzzy supervisory control of a DSP-based magnetic levitation system," Asian Journal of Control, vol. 9, no. 1, pp. 6467, 2007.

[5] S. Banerjee, K. T. K. Sunil, J. Pal, and D. Prasad, "Novel method of controller design for simultaneous stabilization and performance improvement of an electromagnetic levitation system," Asian Journal of Control, vol. 9, no. 4, pp. 402-410, 2007.

[6] H.-J. Shieh, J.-H. Siao, and Y.-C. Liu, "A robust optimal slidingmode control approach for magnetic levitation systems," Asian Journal of Control, vol. 12, no. 4, pp. 480-487, 2010.

[7] K. Ji, W. Kim, and A. Srivastava, "Internet-based real-time control architectures with time-delay/packet-loss compensation," Asian Journal of Control, vol. 9, no. 1, pp. 45-49, 2007.

[8] J. Na, R. Costa-Castelló, R. Griñó, and X. Ren, "Discrete-time repetitive controller for time-delay systems with disturbance observer," Asian Journal of Control, vol. 14, no. 5, pp. 1340-1354, 2012.

[9] B. S. Nesimioglu and M. T. Soylemez, "A simple derivation of all stabilizing proportional controllers for first order time-delay systems," Asian Journal of Control, vol. 14, no. 2, pp. 598-604, 2012.

[10] Y. Ariba, F. Gouaisbaut, and K. H. Johansson, "Robust stability of time-varying delay systems: the quadratic separation approach," Asian Journal of Control, vol. 14, no. 5, pp. 1205-1214, 2012.

[11] H. Wang, J. Li, and K. Zhang, "Sup-resonant response of a nonautonomous maglev system with delayed acceleration feedback control," IEEE Transactions on Magnetics, vol. 44, no. 10, pp. 2338-2350, 2008.

[12] M. Yue and R. Schlueter, "Bifurcation subsystem and its application in power system analysis," IEEE Transactions on Power Systems, vol. 19, no. 4, pp. 1885-1893, 2004.

[13] K. Ben-Kilani and R. A. Schlueter, "An approach for determining the subsystem experiencing and producing a bifurcation in a power system dynamic model," IEEE Transactions on Power Systems, vol. 15, no. 3, pp. 1053-1061, 2000.

[14] A. J. Krener, W. Kang, and D. E. Chang, "Control bifurcations," IEEE Transactions on Automatic Control, vol. 49, no. 8, pp. 12311246, 2004

[15] C. Xia, P. Song, T. Shi, and Y. Yan, "Chaotic dynamics characteristic analysis for matrix converter," IEEE Transactions on Industrial Electronics, vol. 60, no. 1, pp. 78-87, 2013.

[16] L. Yang, Y. Tang, and D. Du, "On Hopf bifurcations in singularly perturbed systems," IEEE Transactions on Automatic Control, vol. 48, no. 4, pp. 660-664, 2003.

[17] W. Zhang and Z. Jiang, "Global Hopf bifurcation in a delayed predator-prey system," in Proceedings of the International Conference on Information, Networking and Automation (ICINA '10), vol. 2, pp. 469-473, October 2010.

[18] Y. Lu, H. Li, and W. Li, "Hopf bifurcation and its control in an induction motor system with indirect field oriented control," in 2009 4th IEEE Conference on Industrial Electronics and Applications, ICIEA 2009, pp. 3438-3441, chn, May 2009.

[19] P. Chen and H. Qin, "Hopf bifurcation stabilization of Rössler system," in Proceedings of the 29th Chinese Control Conference (CCC '10), pp. 452-456, IEEE Conference Publications, July 2010. 
[20] W. Yu, J. Cao, and G. Chen, "Stability and Hopf bifurcation of a general delayed recurrent neural network," IEEE Transactions on Neural Networks, vol. 19, no. 5, pp. 845-854, 2008.

[21] J. Guan and F. Chen, "Hopf bifurcation in Genesio system with delayed feedback," in Proceedings of the 4th International Workshop on Chaos-Fractals Theories and Applications (IWCFTA '11), pp. 8-12, IEEE Conference Publications, October 2011.

[22] K. Natori, R. Oboe, and K. Ohnishi, "Stability analysis and practical design procedure of time delayed control systems with communication disturbance observer," IEEE Transactions on Industrial Informatics, vol. 4, no. 3, pp. 185-197, 2008.

[23] H. Gao, Y. Zhao, and W. Sun, "Input-delayed control of uncertain seat suspension systems with human-body model," IEEE Transactions on Control Systems Technology, vol. 18, no. 3, pp. 591-601, 2010. 


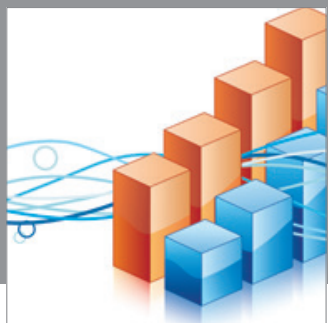

Advances in

Operations Research

mansans

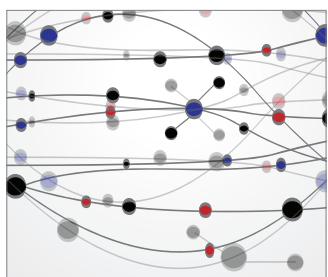

The Scientific World Journal
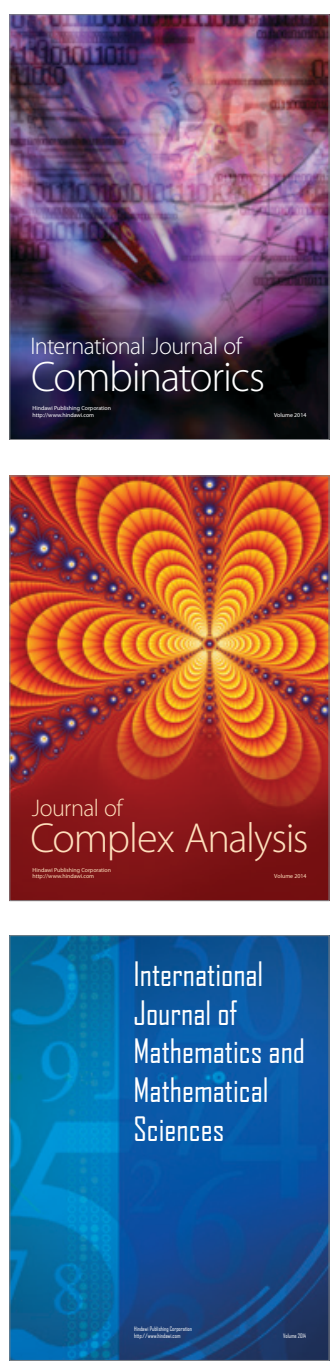
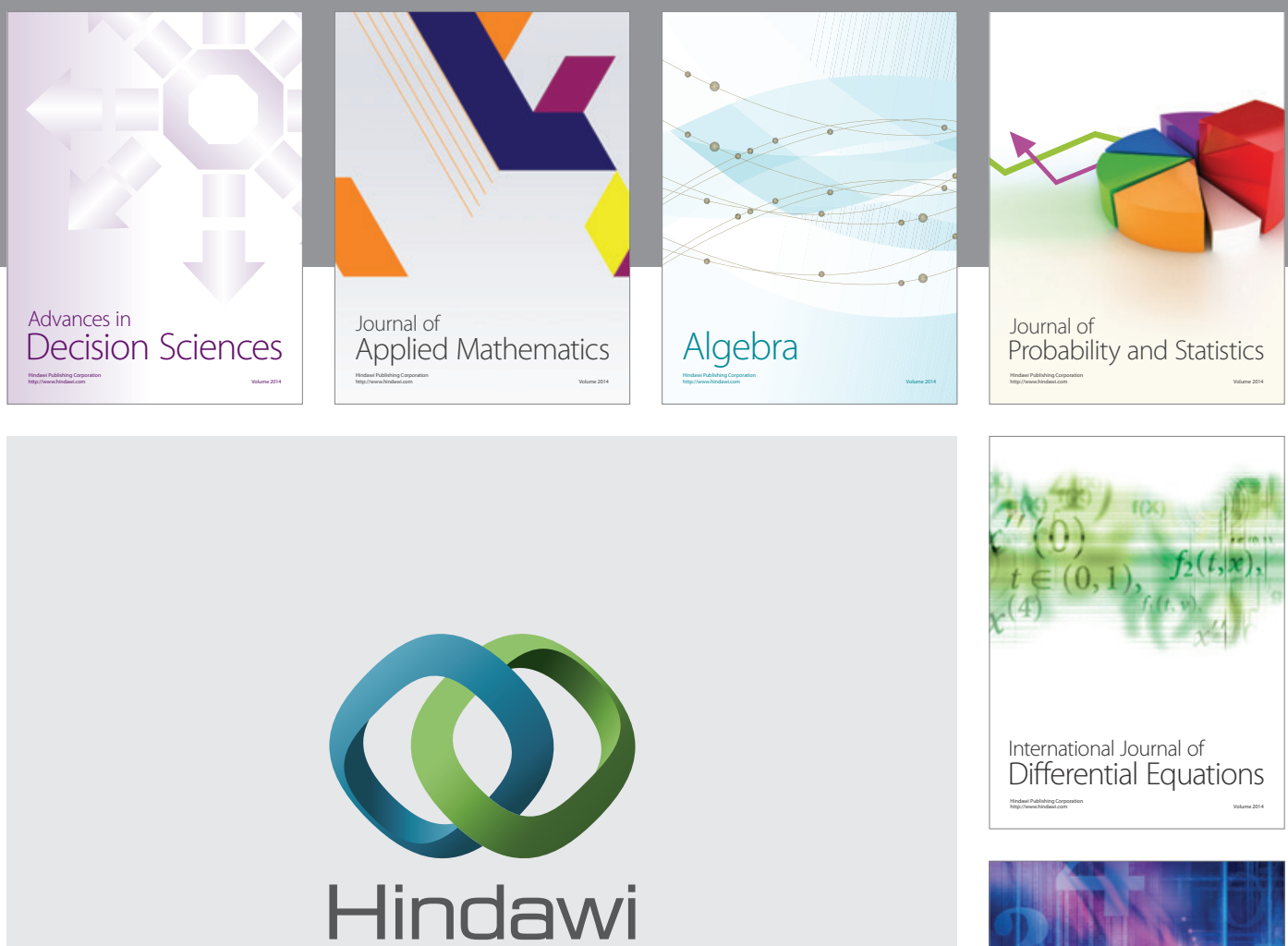

Submit your manuscripts at http://www.hindawi.com
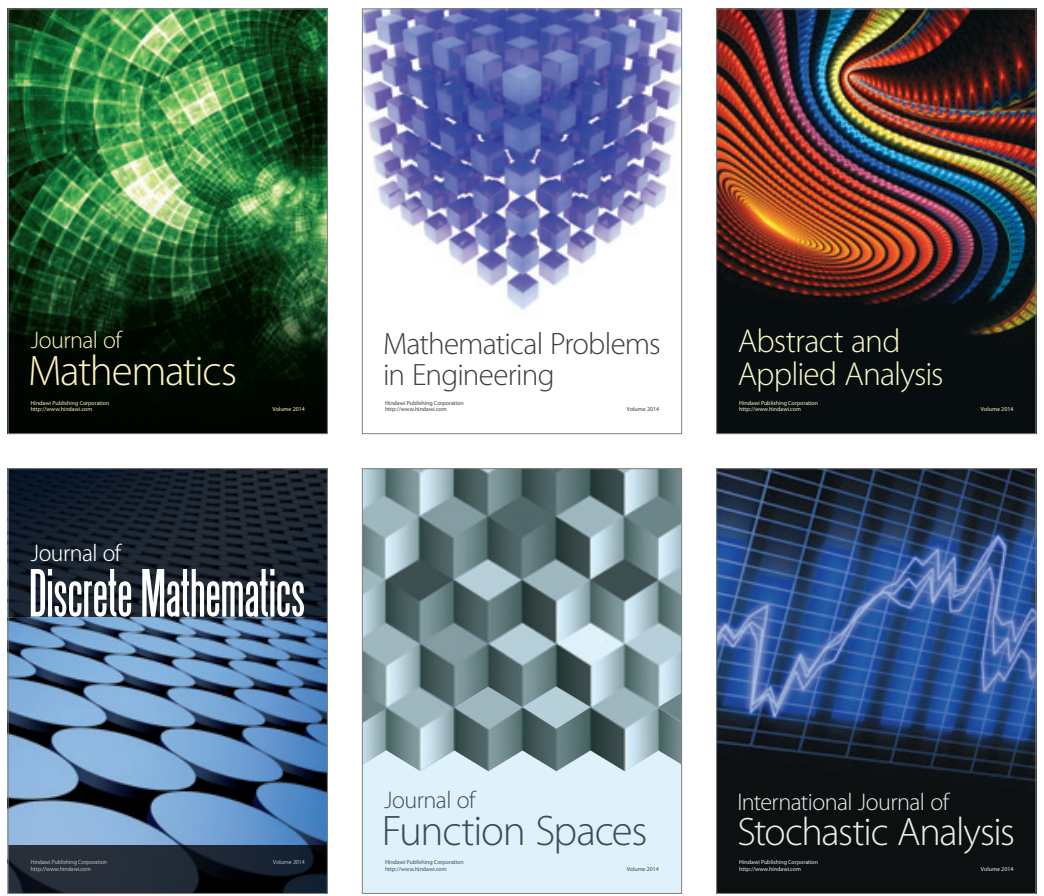

Journal of

Function Spaces

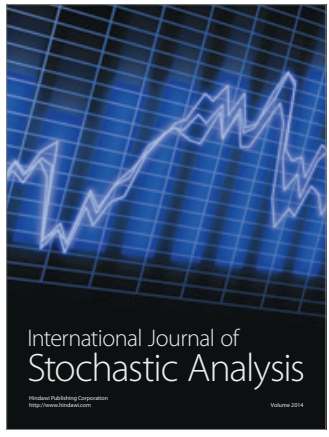

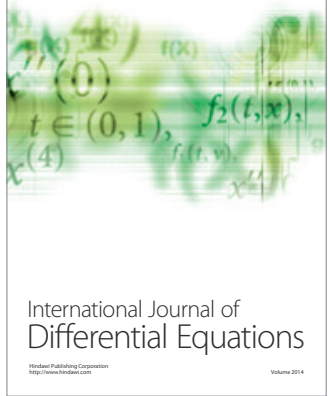
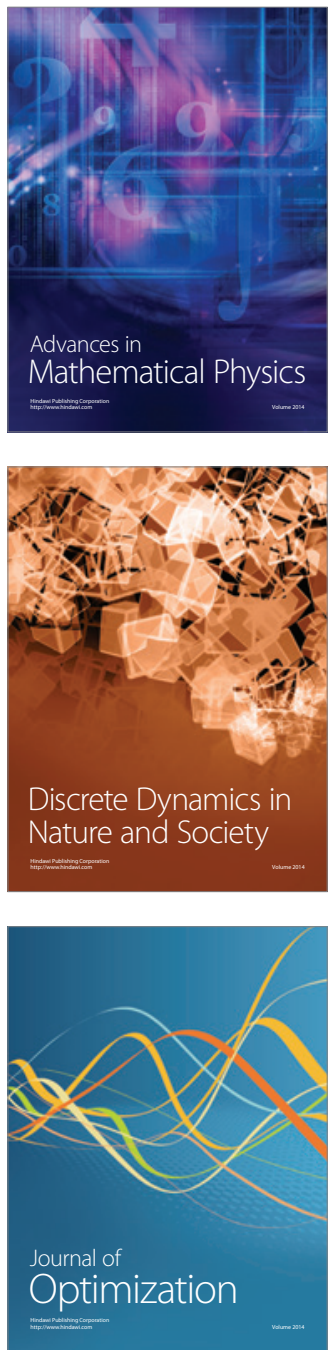